\title{
Frontières
}

\section{Terreur en la demeure}

\section{L'Afrique noire dans l'étau de la guerre civile et du VIH/sida}

\section{Françoise Nduwimana}

Volume 15, numéro 2, printemps 2003

Guerre, mort amère

URI : https://id.erudit.org/iderudit/1073816ar

DOI : https://doi.org/10.7202/1073816ar

Aller au sommaire du numéro

\section{Éditeur(s)}

Université du Québec à Montréal

ISSN

1180-3479 (imprimé)

1916-0976 (numérique)

Découvrir la revue

Citer cet article

Nduwimana, F. (2003). Terreur en la demeure : l'Afrique noire dans l'étau de la guerre civile et du VIH/sida. Frontières, 15(2), 26-31.

https://doi.org/10.7202/1073816ar
Résumé de l'article

La terreur s'est abattue sur l'Afrique subsaharienne durant les deux dernières décennies surtout sous la forme de la guerre civile et de la pandémie du sida. Dans ce contexte éprouvant, on assiste également à une mutation des pratiques de deuil en Afrique noire. Les réformes majeures qui s'imposent nécessiteront une mobilisation des instances internationales.
Ce document est protégé par la loi sur le droit d'auteur. L'utilisation des services d'Érudit (y compris la reproduction) est assujettie à sa politique d'utilisation que vous pouvez consulter en ligne.

https://apropos.erudit.org/fr/usagers/politique-dutilisation/ 


\section{Résumé}

La terreur s'est abattue sur l'Afrique subsaharienne durant les deux dernières décennies surtout sous la forme de la guerre civile et de la pandémie du sida. Dans ce contexte éprouvant, on assiste également à une mutation des pratiques de deuil en Afrique noire. Les réformes majeures qui s'imposent nécessiteront une mobilisation des instances internationales.

Mots clés : Afrique - deuil - sida - guerre.

\begin{abstract}
Terror has ravaged Sub-Saharan Africa for the past two decades especially in the forms of civil war and the AIDS epidemic. Within this harrowing context there is a mutation in the grieving practices of black Africa. The necessary major reforms will require the mobilization of international agencies.
\end{abstract}

Key words : Africa - Mourning - AIDS war.

\section{$\begin{array}{lllllll}A & R & T & \text { I } & C & L & E\end{array}$}

\section{TERREUR EN LA DEMEURE L'Afrique noire dans l'étau de la guerre civile et du VIH/sida}

\author{
Françoise Nduwimana, \\ consultante en droits de la personne \\ et en développement international.
}

Dans son dernier rapport mondial sur le développement humain, le Programme des Nations Unies pour le développement (2002, p. 87) a dressé une liste de 19 pays dans lesquels l'armée était intervenue au cours des années 1990. Il s'agit de l'Algérie, du Burundi, des îles Comores, du Congo, de la Côte-d'Ivoire, de l'Éthiopie, de la Gambie, de la Guinée-Bissau, de Haïti, du Lesoto, de Nyanmar, du Niger, du Nigeria, du Pakistan, de la République centrafricaine, de la République démocratique du Congo, du Rwanda, de la Sierra Leone et de la Somalie. Seulement trois de ces pays ne sont pas africains et, sauf l'Algérie, tous les pays africains qui ont connu un régime militaire au cours des dix dernières années sont localisés au sud du Sahara.

Ce constat est corroboré par Jakob Kellenberger (2001), président du Comité international de la Croix-Rouge et du Croissant-Rouge qui, dans une allocution prononcée le 25 janvier 2001, a affirmé que "parmi les 25 conflits armés les plus importants dans le monde, 11 se déroulaient en
Afrique qui est l'unique région où l'on enregistre une forte augmentation du nombre des conflits armés depuis $1995 »$.

Dans un autre registre, un rapport intitulé Le point sur l'épidémie du SIDA: décembre 2002, publié conjointement par ONUSIDA et l'OMS (Organisation mondiale de la santé, 2002), confirme que l'Afrique subsaharienne demeure la région la plus touchée par la pandémie du sida, avec 29,4 millions de personnes atteintes, sur un total mondial de 42 millions. Alors que le taux de prévalence chez les adultes en Afrique occidentale et centrale (Cameroun, Côte-d'Ivoire, Nigeria, République centrafricaine) se situe entre $10 \%$ et $15 \%$, l'Afrique australe (le Bostwana, le Lesotho, le Swaziland, le Zimbabwe, etc.) atteint des niveaux inimaginables avec plus de $30 \%$ des adultes porteurs du VIH.

Derrière toutes ces statistiques se trouve une Afrique endeuillée, dépouillée de quelque vingt millions d'âmes enlevées par le sida. Une Afrique écorchée, qui voit languir plus de trente millions de séropositifs dans le couloir de la mort. Une Afrique à la mémoire blessée, au présent en sursis et à l'avenir hanté. Une Afrique fortement marquée par la terreur. Terreur engendrée par 
la violence des conflits armés qui ont fait, en Angola, 500000 morts, 4 millions de déplacés et 100000 mutilés (Geslin, 2002) ; à la Sierra Leone, 75000 morts, 500000 réfugiés, et 4,5 millions de déplacés (Smillie, Gberie et Hazleton, 2000) ; en République démocratique du Congo, 1,7 million de morts et 1,3 million de déplacés (Tougas, 2000).

Au regard de ce tableau, on serait tenté de trouver normal que le continent africain, tel qu'il est dépeint par les médias, offre aujourd'hui la triste image d'un continent fragmenté, qui se livre à l'autodestruction. En effet, tout panorama construit dans une logique et dans une culture du sensationnel et de l'instantané ne peut pas permettre le recul et la remise en contexte que requiert toute analyse de fond.

Dès lors qu'on appréhende des crises aussi profondes non pas en vue d'en connaître les causes et les conséquences, mais simplement dans le but de montrer ce qui se passe ailleurs, le risque est grand de voir les victimes transformées en simples statistiques. Le risque est aussi grand de cultiver l'inaction à travers le caractère préalablement déclaré inextricable de ces crises. En ignorant les tenants et les aboutissants de ces situations, on réduit considérablement les possibilités de provoquer un ébranlement collectif susceptible d'amorcer une démarche de résistance et de changement durable et solidaire.

La terreur s'est abattue sur l'Afrique subsaharienne durant les deux dernières décennies. À travers ces deux défis majeurs de l'Afrique contemporaine que sont la guerre civile et la pandémie du sida, nous allons décrire la manière dont la vie des peuples au sud du Sahara est réduite à la survie. Par terreur, nous entendons aussi une réalité psychologique, celle de la détresse omniprésente. Cette terreur meuble l'imaginaire des enfants orphelins du sida et de la guerre. Des enfants en rupture d'enfance. Elle terrasse la femme dans ce qu'elle a de plus intime, son corps et sa sexualité, car canal de vie, le corps de la femme victime de violence devient aussi canal de mort.

Le choix de ces deux défis a été dicté par trois constats. Premièrement, il va sans dire, d'une part, que les deux phénomènes constituent une sérieuse menace à la stabilité et à la sécurité de l'Afrique et que, d'autre part, ils se nourrissent mutuellement, tout en exploitant le même terreau fertile, soit la pauvreté.

Même les ombres portées par le soleil tracent l'enfermement.
En s'imposant pratiquement comme une activité lucrative, la guerre civile exploite ce fond de la pauvreté. Le phénomène des enfants soldats en est la parfaite illustration. Le trafic des armes et des matières premières comme c'est le cas à la Sierra Leone et en République démocratique du Congo, montre à quel point, à défaut d'autres horizons, toute une génération d'enfants africains est galvanisée vers le crime et l'enrichissement personnel, renonçant ainsi à jouer le rôle de bâtisseur d'avenir que la société attend d'elle. Mais la guerre n'exploite pas uniquement la misère, elle la radicalise aussi. Elle conduit à l'effondrement des structures sociales, elle est source de violence physique et sexuelle, d'insécurité et d'instabilité. La guerre produit des réfugiés et des déplacés. La guerre est aussi un important vecteur du VIH / sida.

Dans la même logique, il n'y a pas l'ombre d'un doute que la pauvreté extrême porte les germes du VIH / sida. Ce fléau a fait son apparition au moment où l'Afrique était sommée d'adopter les plans d'ajustement structurel. Basés sur une logique d'assainissement des finances publiques, ces plans ont eu pour effets la privatisation ainsi que le sous-financement des secteurs névralgiques comme la santé et l'éducation.
Il s'en est suivi une déconfiture sociale accentuée par le paiement de la dette extérieure et par la chute de l'aide publique au développement. Dans une telle situation, où la moitié de la population survit avec un revenu journalier inférieur à $1 \$$, comment s'étonner que l'espérance de vie en Afrique $^{1}$ soit de 49 ans, alors qu'elle est de 78 ans dans les pays développés?

Deuxièmement, la guerre et le sida ont permis de dévoiler l'enjeu du statut de la femme africaine. Car si ces deux fléaux hypothèquent l'avenir du continent, ils prennent à partie, de façon ciblée et particulière, la femme africaine. C'est ce que révèlent les données d'ONUSIDA publiées le $1^{\text {er }}$ décembre 2002, Journée mondiale de lutte contre le sida, qui indiquent que les femmes africaines constituent $58 \%$ des personnes atteintes. C'est aussi ce que révèle le Haut Commissariat des Nations Unies pour les réfugiés qui, dans son bulletin Refugees (2002), estime que 75 à $80 \%$ des réfugiés sont des femmes et des enfants.

L'absence de pouvoir chez les femmes (voir Nduwimana, 2001), que ce soit en matière de contrôle de la sexualité ou en matière politique et économique, et la non-reconnaissance de leur statut expliquent pourquoi elles sont les principales

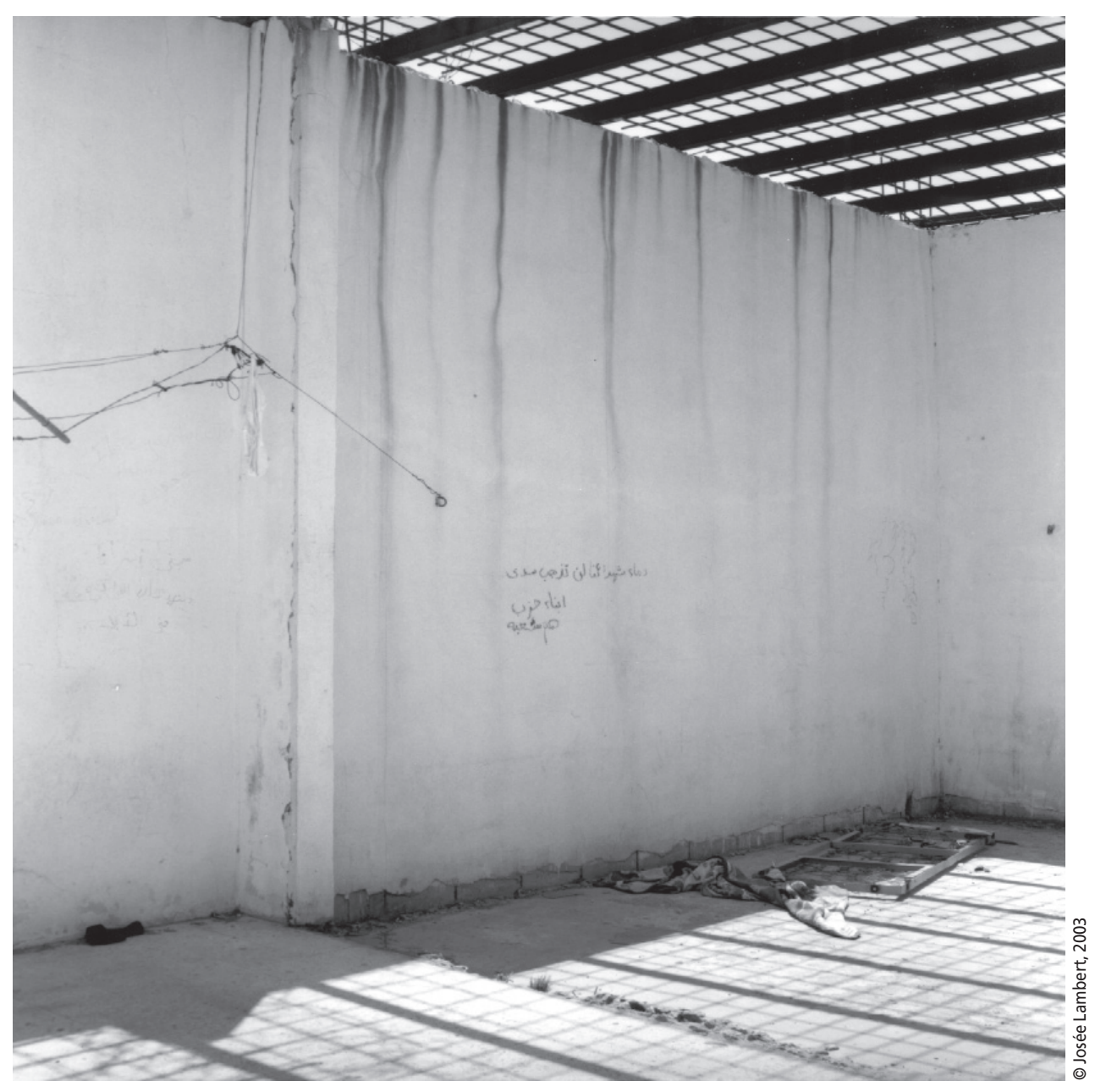


victimes, mais aussi pourquoi l'Afrique est privée de leurs ressources et de leur force dans la recherche de solutions durables. Autrement dit, si les femmes africaines pouvaient vraiment exercer leurs droits sociaux, économiques, culturels et politiques, elles interviendraient de façon plus substantielle dans la stabilité et la sécurité du continent, pour le bénéfice de toute l'Afrique.

Enfin, plusieurs visites effectuées dans les Grands Lacs nous poussent à réaliser que par la façon terrifiante dont ils anéantissent la vie, par l'ampleur des pertes humaines et de la détresse qu'ils engendrent, la guerre et le sida sont en train de reconfigurer complètement le rapport africain au deuil et à la mort. Si le respect de la mort reste jalousement gardé par la famille éprouvée, le deuil par contre ne se vit plus du tout de la même façon.

L'épuisement moral et physique des familles ainsi que l'incapacité à assurer le coût financier des obsèques quasi journalières ont eu raison des fondements et rituels sur lesquels reposait le deuil africain. Il s'agit d'une mutation lourde de signification. En un laps de temps insignifiant du point de vue historique, soit vingt ans, le phénomène du sida et de la guerre ont chamboulé des siècles et des siècles de civilisation.

\section{LA MORT, SON SENS, SA RECEVABILITÉ}

La mort demeurera toujours, pour l'humain, une expérience douloureuse et mystérieuse. Douloureuse parce qu'elle emporte à jamais ceux et celles qui confèrent une solide fondation à la vie, ceux et celles pour qui nous vivons, à travers et grâce à qui nous nous définissons. Le destin de l'humain ne se trace pas à l'ombre, mais à l'aune de son semblable.

Même dans les sociétés les plus individualistes, l'homme et la femme sont des êtres d'histoire, de communauté et de continuité. Communauté et histoire parce que, comme le veut la sagesse africaine, nous héritons d'un monde qui nous a précédés. Nous ne sommes qu'une calebasse au fil de l'eau. Continuité parce que notre histoire se tisse à travers nos progénitures, à travers ceux et celles à qui nous aurions eu le devoir de confier le monde. C'est au bout de la vieille corde, dit un proverbe africain, qu'on tisse la nouvelle.

Mais la mort a ceci de mystérieux qu'elle fait partie intégrante d'une dynamique siamoise et dualiste, vie-mort. Et tout compte fait, si elle enlève la vie, elle la définit aussi. De la même manière que nous nous projetons à travers les yeux, les parcours et l'existence de nos semblables, nous définissons le bonheur ou la tristesse de la vie, non

LA GUERRE ET LE SIDA TERRORISENT ET MATRAQUENT PSYCHOLOGIQUEMENT DES VILLAGES ENTIERS QUI VOIENT DISPARAITTRE, PRÉCOCEMENT, LEURS FORCES VIVES.

seulement au reflet de la mort qui nous est encore épargnée, mais aussi à la lumière des souvenirs laissés par les disparus.

Dans plusieurs sociétés africaines, le soin que l'on met à choisir les mots ou les figures utilisés pour désigner la mort est révélateur de la vénération qu'on voue à cette expérience humaine. Départ, passage, visite de l'au-delà, appel divin, sommeil, etc., sont couramment choisis à la place de décès à proprement parler. Cette façon d'appréhender la mort est antérieure à l'arrivée du christianisme et de l'islam, elle s'inspire des traditions et spiritualités africaines. Du point de vue anthropologique, la vénération de la mort révèle une profonde conviction selon laquelle les morts continuent à habiter le monde et constituent en quelque sorte la pierre angulaire du pacte de la vie et de la mort. Il suffit de consulter l'anthologie noire pour s'en rendre compte. Dans son célèbre poème Souffles, le Sénégalais Birago Diop, poète de la lignée de la Négritude, figure de proue de la littérature francoafricaine $\mathrm{du} \mathrm{XX}^{\mathrm{e}}$ siècle, ne révèle-t-il pas la présence mystique des disparus?

\section{Écoute plus souvent}

Les Choses que les Êtres,

La Voix du Feu s'entend,

Entend la Voix de l'Eau,

Écoute dans le Vent

Le Buisson en sanglots:

C'est le Souffle des Ancêtres.

Ceux qui sont morts

ne sont jamais partis :

Ils sont dans l'Ombre qui s'éclaire

Et dans l'Ombre qui s'épaissit.

Les Morts ne sont pas sous la Terre:

Ils sont dans l'Arbre qui frémit,

Ils sont dans le Bois qui gémit,

Ils sont dans l'Eau qui coule,

Ils sont dans l'Eau qui dort,

Ils sont dans la Case,

ils sont dans la Foule:

Les Morts ne sont pas morts.[...]

Ils sont dans le Sein de la Femme,

Ils sont dans l'Enfant qui vagit

Et dans le Tison qui s'enflamme [...]

Il redit chaque jour le Pacte,

Le grand Pacte qui lie

Qui lie à la Loi notre Sort, [...]

Le lourd Pacte qui nous lie à la Vie. [...]

(dans Warner, 1971, p. 86-88).
Selon le poète, nous respirons ce souffle et le monde qui nous entoure est à la fois un lieu de mémoire et une demeure où les vivants et les morts ne font qu'un.

Plus proche du Québec, Émile Ollivier (Ollivier, 1991, p. 100), disparu mais toujours vivant, dit dans Passages : "Je n'ai pas besoin de vous rappeler, monsieur, l'importance des morts chez nous. Vous savez aussi bien que moi qu'ils sont présents, nous accompagnent dans tous les gestes de la vie. Ils sont notre lumière et non nos ténèbres ou plutôt, ils sont la lumière qui éclaire nos ténèbres. »

Toutefois, si à travers le culte de vénération de la mort les traditions noires acceptent et épousent en quelque sorte cette réalité, cela ne veut pas dire qu'il n'y a pas de limite à cette forme d'adoration. Le culte de la mort ne signifie pas une abdication devant la mort. Il vise plutôt à apprivoiser la mort, à l'aborder et à l'exorciser. Il permet aux vivants de ne pas perdre le contrôle devant ce mystère. Il vise à trouver des façons plus symboliques de composer avec une réalité qui les dépasse. Il vise à protéger la vie après la mort, donc à protéger la mémoire. Loin de favoriser la passivité devant la mort, le culte encadre la recevabilité de la mort.

Ainsi, si la mort, de façon générale, fait partie de l'expérience humaine, la mort "gratuite », c'est-à-dire celle qu'on peut éviter et qu'on aurait pu éviter, est inacceptable, peu importe qu'elle survienne au Nord, au Sud, à l'Est ou à l'Ouest. Alors qu'ils ont presque été éradiqués dans des pays riches, la guerre et le sida continuent d'être des entraves à la vie dans des pays pauvres, en Afrique en particulier.

Prise entre ces deux feux, l'Afrique noire apparaît comme un sous-continent sous l'emprise de l'hypnose, entraîné dans une danse macabre dont le duo est composé du sida et de la guerre. Cette figure de duo macabre est tellement ancrée dans l'imaginaire qu'elle transparaît automatiquement dans toutes les formes d'expression, que ce soit l'art, la chanson populaire, le théâtre, la littérature ou la danse.

C'est ainsi qu'abordant le défi de la paix dans la région des Grands Lacs africains, Nathalie Gisabo Gahunga, écrivaine et représentante de la Concertation des collectifs d'associations féminines de la région des Grands Lacs africains, y associe spontanément le défi du VIH / sida. "Au sud, la 
situation conflictuelle, la pandémie du sida, la commercialisation des armes et les alliances des gouvernements au gré de leurs intérêts économiques constituent des menaces à la paix », écrit-elle (Gahunga, 2001, p. 23).

Dans la même lignée, décrivant l'horizon funèbre qui enveloppe le sous-continent, Joseph Arimoso, Jésuite du Zimbabwe, lance un cri du cœur dans son poème «Sur le SIDA en Afrique » où l'on décèle l'impuissance devant l'indescriptible:

Un vent noir hurle à travers la terre porteuse de malédictions

Quel est ce vent qui porte en lui d'incontrôlables chants funèbres?

Le fruit tendre tombe sur le sol, trop vert et le vent sombre l'emporte dans la blessure béante de la terre

La terre verdoyante n'est plus, écorchée de douleur

Monceaux de terre qui surgissent dans des champs riches d'ossements

Alors que les âmes rebelles dorment dans une quiétude impuissante

Le tambour triste n'en peut plus de gémir [...] (Arimoso, 2002).

Encore plus révélatrices, les enquêtes internationales sur les méfaits de la guerre ne peuvent plus passer sous silence l'association de la guerre avec la pandémie du sida. C'est ce qui ressort du rapport intitulé La guerre dans la guerre. Violence sexuelle contre les femmes et les filles dans l'est du Congo, rapport d'enquêtes internationales publié par Human Rights Watch en juin 2002, qui pousse à se demander si le degré de barbarie exercée sur les femmes par le canal de la guerre et du sida ne sonne pas le glas de l'essence féminine? «Naître fille dans ce pays ne signifie-t-il pas être condamnée à mort dès la naissance ? », y lit-on à la page 36 .

Le roman n'y échappe pas. Si le livre de Gilles Courtemanche, Un dimanche à la piscine à Kigali, publié chez Boréal en 2000, a plusieurs mérites, tous n'ont pas été soulignés par la critique. L'un des traits importants de ce roman est justement d'avoir su créer cette atmosphère de suicide collectif qui a prévalu au Rwanda avant et durant le génocide, et dans laquelle la violence liée à la guerre et à la pandémie du sida s'est déployée tel le tandem de l'infâme.

Le sexe y fait figure d'instrument de violence et de pouvoir. Il est étalé dans toute sa grossièreté. Chose d'autant plus intrigante qu'au Rwanda, comme dans plusieurs pays africains, le sexe est tabou au niveau du discours. Le rapport au sexe est marqué par le tabou de la parole directe. Au Rwanda et au Burundi, où le langage imagé ainsi que les figures de style ont préséance sur le langage direct, la sexualité, domaine de l'intime et du privé par excellence, n'est jamais ouvertement et directement évoquée. Ce rapport au sexe a rendu difficile la politisation de certains enjeux dont le planning familial, la violence sexuelle et la prévention au VIH / sida.

Mais cette culture du tabou du sexe est aussi le fruit du contrôle moral exercé par l'Église catholique sur les structures étatiques. Comme l'a souligné une spécialiste du Rwanda, Colette Braeckman, c'est particulièrement dans les domaines de la contraception et de la prévention du VIH qu'un tel pouvoir s'est manifesté (1994, p. 96-97). Les institutions rwandaises, sous la pression des évêques et des groupes charismatiques, n'ont eu d'autres choix que de renoncer à la promotion du préservatif et de la pilule.
Le deuxième trait qui est lié au premier mais qu'il est aussi important de souligner concerne la gravité des crimes qui sont couramment commis dans le cadre de la guerre civile. Ils sont d'une extrême violence. Appuyée sur une logique d'extermination de l'ennemi comme si on voulait exterminer la vermine, la guerre civile est le meilleur terrain des massacres collectifs, assassinats, mutilations, viols, tortures, disparitions, déplacements forcés, etc. Si, en jargon juridique, on appelle ces crimes, des crimes de guerre ou des crimes contre l'humanité, un tel degré de barbarie est tout simplement innommable.

Le troisième trait de la guerre civile en Afrique est sa longue durée. Rares sont les cas où elle dure moins de dix ans. Même si

CETTE PRISE EN CHARGE DES MALADES EST ASSUMÉE

PAR LES AIDANTES NATURELLES QUE SONT LES FEMMES. LA MORT SURVIENT ALORS QUE L'ÉPUISEMENT EST À SON COMBLE. ELLE SURVIENT AU MILIEU D'UNE TERRIBLE QUESTION: QUEL RÉPIT ENTRE CE DEUIL ET LE SUIVANT ?

\section{MUTATION DU DEUIL}

Pour comprendre la mutation que le deuil en Afrique noire a subie, il est utile et nécessaire de remonter aux caractères singuliers que revêtent deux des plus importantes causes de la mort massive sur ce continent, la guerre civile et la pandémie du sida.

La guerre en Afrique est atypique. Elle comporte plusieurs aspects propres à l'histoire africaine et qui expliquent la façon, unique, dont elle affecte le tissu social, au point de pervertir le rapport au deuil. Plus qu'un conflit armé, la guerre en Afrique est une guerre civile, un conflit interne qui voit les hostilités se dérouler à l'intérieur des frontières nationales. À quelques exceptions près, on pourrait dire que c'est une guerre fratricide qui oppose les composantes d'une même nation. Ici, la logique de la solidarité sanguine prend facilement le pas sur la confrontation des projets de société.

Dans ce genre de guerre où, d'après le PNUD, $90 \%$ des victimes sont issues de la population civile, est déclarée ennemie à abattre toute personne du camp adverse, c'est-à-dire celle dont on ne partage pas le giron politique, ethnique, clanique, religieux, régional, etc. Dans un tel contexte, où seule la force a droit de cité, les plus faibles, les enfants, les femmes, les vieillards sont sacrifiés au grand dam du droit humanitaire international. certains d'entre eux émergent des conflits armés, les pays comme le Mozambique, l'Angola, l'Ouganda, le Rwanda, le Burundi, la République démocratique du Congo, la Somalie, le Soudan, le Libéria, la Sierra Leone, l'Algérie, ont tous en commun plus de dix ans de guerre civile. À ces pays, il faut ajouter une multitude de crises politiques récurrentes qui constituent des bombes à retardement. C'est le cas de la Côte-d'Ivoire, de la République centrafricaine, de la République populaire du Congo, du conflit entre l'Éthiopie et l'Érythrée, etc.

De son côté, le sida est une maladie que l'Afrique héberge depuis deux décennies. C'est aussi une maladie qui, avant d'achever ses victimes, peut s'avérer longue. Dans ce cas de non-accès aux médicaments qu'est celui de l'Afrique noire, il n'y a pas d'autre perspective que la mort. Les malades, avant de rendre l'âme, ont le temps de se décomposer dans ce qu'il convient d'appeler le couloir de la mort.

Comme la guerre, la terreur engendrée par le SIDA n'est plus à démontrer. D'abord il $\mathrm{y}$ a le nombre effarant des personnes infectées et affectées, celui des personnes décédées et celui de prochaines victimes. Ensuite il y a aussi l'âge et le sexe des victimes; de plus en plus de jeunes, de plus en plus de femmes. Mais le sida est aussi indicible dans sa façon, unique, de 
transmettre la mort, par les symboles mêmes de la vie, ceux par qui la vie est donnée, le sexe, le sang et le lait maternel.

Les deux phénomènes expliquent comment, du vénéré et riche rituel célébrant la mémoire, le deuil en Afrique, quand il est permis, est réduit à une formalité dont on a vite envie de s'acquitter. Dans ce bout du monde, il y a trop de morts, trop de victimes innocentes, depuis trop longtemps. Cela a dénaturé le deuil. Au fond, c'est quoi un deuil si ce n'est la capacité et la possibilité de méditation, de recueillement, de recul par rapport à la vie ? La mémoire des disparus, ce fameux pacte de la vie et de la mort, ne peut se cultiver que lorsque la douleur de perdre est atténuée par l'espoir des lendemains qui chantent.

C'est ici que le bât blesse. Plusieurs nations africaines vivent dans une culture mortifère. La mort massive meuble l'imaginaire. Dans une telle circonstance où pendant qu'on enterre, l'attention est portée non plus sur la personne décédée mais sur ce qui attend les suivants, le deuil perd son sens premier, celui de distance face à l'immédiat, celui du retour en arrière, vers les souvenirs.

Dans plusieurs traditions africaines, particulièrement dans les anciens royaumes interlacustres, la période du deuil observé à l'occasion du décès d'un adulte s'allonge sur une année. Mais seulement le premier mois est à proprement parler actif. Ainsi, dès l'annonce du décès, la dépouille mortelle est placée au salon de la demeure familiale pour que les proches fassent une dernière veillée en sa compagnie. Cette veillée est l'occasion de parler du défunt ou de la défunte. C'est aussi l'occasion de soutenir moralement la famille éprouvée. Pendant la veillée, chants, incantations et prières alternent jusqu'à la tombée du jour. Viennent alors les obsèques. C'est une occasion où tous les amis du quartier, du village, tous les membres proches ou éloignés de la famille se retrouvent pour un dernier hommage. On entre alors dans la semaine du deuil durant laquelle la famille reçoit visites, présents et réconfort de façon substantielle. À la fin de cette semaine, une cérémonie de levée de deuil partielle est organisée afin de permettre le retour au travail. Le mouvement des visites et présents se poursuit généralement pendant un mois. Après il devient de plus en plus espacé. Enfin, une année après le décès, une grande cérémonie de levée de deuil définitive est organisée. Il appartient alors à la famille et aux amis d'entretenir la mémoire à leur rythme et convenance.

Cette façon de faire et de vivre le deuil tend à disparaître, tellement les aléas de la guerre et du sida imposent non seulement leurs propres limites, mais surtout anéan-

tissent le droit de vivre le deuil avec sérénité. Dans une situation de conflit armé, il arrive que la date et l'heure de l'enterrement ne soient pas déterminées par les familles mais par les conditions de sécurité. Et nombreux sont les cas où l'insécurité empêche carrément la présence des membres de la famille vivant loin. Face à la guerre, il est courant qu'en cas de massacres collectifs, les victimes soient jetées dans des fosses communes par leurs bourreaux et que les familles ne mettent jamais la main sur leurs dépouilles. Il arrive aussi qu'en cas de disparitions ou d'exécutions extrajudiciaires, les familles demeurent dans le doute. Or le processus du deuil suppose la certitude du décès, il suppose la disposition de la dépouille. Pire que la douleur de perdre, l'impossibilité d'accompagner les disparus est on ne peut plus dévastatrice.

\section{SI LA COMMUNAUTÉ INTERNATIONALE CESSAIT DE RÉDUIRE LA PAIX} ET LA SÉCURITÉ DU MONDE À LA PAIX ET LA SÉCURITÉ DU NORD. L'OCCIDENT N'EST PAS LE MONDE. IL EN FAIT PARTIE. PAR CONTRE, SA STABILITÉ DÉPEND DE CELLE DU RESTE DU MONDE.

Le défi du deuil lié au VIH / sida comporte les mêmes embûches. Comment vivre un deuil avec sérénité quand l'horizon est funèbre? Où trouver la force quand l'avenir est rythmé par l'ombre de la mort? Où trouver le support moral quand sur tout le monde plane l'épée de Damoclès? Comment rendre un hommage personnalisé quand il y a autant de décès quotidiens, et autant de vies en sursis? Face à la détresse de la mort, il y a aussi l'essoufflement moral et l'épuisement physique des familles. Car en contexte africain où, sous le diktat des politiques néolibérales, l'État-providence, déjà embryonnaire, a été démantelé sans autre forme de procès, la prise en charge des malades, celle des orphelins incombe aux familles et aux communautés (voir Nduwimana, 2002). Autrement dit, cette prise en charge des malades est assumée par les aidantes naturelles que sont les femmes. La mort survient alors que l'épuisement est à son comble. Elle survient au milieu d'une terrible question: quel répit entre ce deuil et le suivant?

On ne peut donc pas s'étonner que le deuil africain soit en passe de devenir anodin. Pour gagner du temps, les Églises, elles-mêmes essoufflées, favorisent des messes collectives où on célèbre plusieurs décès à la fois. Pis encore, de la messe dédiée aux défunts, on passe de plus en plus à une simple bénédiction des dépouilles.
Parce qu'elles sont devenues trop coûteuses, les réceptions qui accompagnent les enterrements et les levées de deuil ne sont plus considérées comme une occasion de célébration, mais bien plus comme une obligation au coût financier et humain considérable. Ces cérémonies sont de plus en plus réduites au minimum, au partage d'un verre. Rares sont les familles qui attendent un an avant de décréter la fin du deuil. De plus en plus, la levée de deuil partielle sert d'occasion pour décréter la levée de deuil définitive. Devant le nombre élevé de décès, les familles sont à court d'énergie.

On voit çà et là, l'arrivée des entreprises de pompes funèbres. Pour les mieux nantis, ces entreprises offrent un répit. Dans le même temps, elles sonnent le glas du pacte entre les morts et les vivants. Car en offrant les services liés aux obsèques, les pompes funèbres interfèrent dans le complexe rituel qui régit le deuil. Désormais, la mort est une affaire très lucrative. Désormais le mort est confié à des inconnus, ceux des pompes funèbres ; du jamais vu dans l'histoire de l'Afrique noire!

\section{NI LA GUERRE, NI LE SIDA NE SONT DES CATASTROPHES NATURELLES}

L'ampleur des pertes humaines et du désordre social peut pousser l'observateur à faire de l'Afrique un continent banni. Néanmoins, il faut déconstruire le caractère inné qu'on impute à la guerre africaine et à la propagation exponentielle du sida. Si le sida fait autant de ravages en Afrique, c'est en vertu de plusieurs facteurs dont la pauvreté chronique qui y sévit et qui constitue en quelque sorte un élément d'amplification. De même, si l'Afrique est un continent dominé par les conflits armés, on ne peut pas ignorer que ceux-ci sont en partie le prolongement des crises incubées dans le terreau colonial, mais aussi le fruit d'une gabegie dont plusieurs conglomérats étrangers tirent profit.

Les exemples de la région des Grands Lacs, du Liberia et de la Sierra Leone sont assez éloquents. Pour les Grands Lacs, d'aucuns s'accordent pour souligner le rôle joué par la Belgique, ancienne puissance 
coloniale, dans la création et l'entretien de la rivalité des identités ethniques (voir Chrétien, 1997 ; Braeckman, 1996). Par ailleurs, un rapport d'experts de l'ONU sur le pillage des ressources minières a démontré l'implication de 85 compagnies étrangères dont les trois-quart sont enregistrés en Amérique du Nord et en Europe de l'Ouest (voir Khan, 2002). Pour ce qui est du Liberia et de la Sierra Leone, d'une part, il va sans dire que l'un des écueils de l'unité nationale réside dans le fait que ces deux pays ont été promis aux esclaves affranchis sans que les autochtones aient eu leur mot à dire (Smillie, Gberie et Hazleton, 2000). D'autre part, il a été prouvé que l'enjeu de cette guerre est étroitement lié à la réalisation de bénéfices provenant d'activités minières et que les compagnies diamantifères comme De Beers et le Diamond High Council ont été compromises dans la violence liée à l'extraction du diamant.

Peut-on alors moralement abandonner l'Afrique, arguant que sa stabilité est une affaire interne? La paix en Afrique est possible. Mais elle n'est pas uniquement une affaire africano-africaine. Elle concerne aussi bien les actes criminels commis au nom de la colonisation que ceux commis au nom de la dictature africaine. Le sentier de la paix africaine passe par un devoir de solidarité et de mémoire envers l'Afrique, par l'arrêt du commerce illicite des armes, par l'arrêt du pillage des ressources minières africaines, par l'arrêt du soutien aux despotes africains.

Dans la même logique, si le sida reste une maladie incurable, son éradication est possible grâce à des mesures de prévention et d'allongement de l'espérance de vie des malades. Ceci exige beaucoup de moyens en termes d'argent, de ressources humaines et d'infrastructures sociales. Ces moyens qui, sous d'autres cieux, ont permis de contrôler la propagation du VIH / sida, font cruellement défaut à l'Afrique.

Enfin, il est important de noter que la mort est discriminatoire à l'égard des femmes. Une femme qui voit sa vie détruite par une violence délibérée est forcément une victime préméditée. Ce fut le cas du Rwanda où, d'après l'ancien rapporteur spécial de l'ONU pour le Rwanda, René Dégni-Ségui, entre 250000 et 500000 femmes auraient été violées durant le génocide. Ce fut aussi le cas de la Sierra Leone où, d'après Physicians for Human Rights (2002), 53 \% des femmes et des filles déplacées, soit 65000 femmes, auraient été victimes des violences sexuelles.

La quête d'égalité que revendique le féminisme africain n'est pas une chimère. Elle concerne autant le droit à la terre, à la participation que le droit au leadership. L'éducation, la santé, le pouvoir politique et économique sont des conditions sans lesquelles la sécurité et la dignité des femmes ne peuvent s'exercer.

\section{ENJEUX ET PERSPECTIVES}

Le sida et la guerre ont mis en exergue plusieurs maux dont l'Afrique souffre depuis les deux dernières décennies. Ces deux crises devraient amorcer un débat de fond sur quelques enjeux majeurs dont les causes historiques et structurelles des conflits armés, celles ayant engendré la pauvreté, et le déficit démocratique.

Le sida et la guerre devraient pousser à une réflexion sur le devoir de mémoire et de solidarité envers l'Afrique, au moment où le commerce international est posé comme le principal point d'ancrage en dehors duquel il est impossible de penser l'entraide internationale.

Le sida et la guerre sont certes des catastrophes humanitaires, mais elles ne sont pas immuables. Se transformant en urgences permanentes, elles soulèvent une question morale, soit l'acceptation par l'humanité de la souffrance continue de l'Afrique noire. Ces deux fléaux peuvent être éradiqués si la bonne gouvernance démocratique s'implantait dans les mœurs politiques et si la communauté internationale cessait de réduire la paix et la sécurité du monde à la paix et à la sécurité du Nord. L'Occident n'est pas le monde. Il en fait partie. Par contre, sa stabilité dépend de celle du reste du monde.

Enfin, des réformes majeures sont requises si on veut vraiment réhabiliter le statut de la femme. En temps de sida et de guerre, le droit pénal international ne peut plus faire fi de la situation de condamnée à mort dans laquelle la femme violée par un porteur du VIH se retrouve. La justice, si justice il y a, si elle n'impose pas des mesures de réparation comme le droit d'accès aux médicaments, aura raté l'une de ses principales missions : reconstruire les vies brisées.

Pourquoi ne pas suivre l'exemple de l'Afrique du Sud, qui n'est pas un cas de guerre civile mais de violence généralisée illustrée par un viol toutes les 26 secondes, où les tribunaux ont tranché en faveur des femmes violées, enjoignant le gouvernement de leur fournir des médicaments gratuits? L'absence d'une telle disposition au niveau du droit pénal international constitue une lacune que les législateurs sont appelés à corriger de toute urgence.

\section{Bibliographie}

ARIMOSO, Joseph (2002 [2001]). «Sur le SIDA en Afrique", Hekima Review, décembre 2001, traduit en français par Christine GAUTHIER et repris dans Headlines de mars 2002.

BRAECKMAN, Colette (1996). Terreur africaine. Burundi, Rwanda, Zaïre: les racines de la violence, Paris, Fayard.

BRAECKMAN, Colette (1994). Rwanda. Histoire d'un génocide, Paris, Fayard.

CHRÉTIEN, Jean-Pierre (1997). Le défi de l'éthnisme, Karthala.

COURTEMANCHE, Gilles (2000). Un dimanche à la piscine à Kigali, Montréal, Boréal.

GAHUNGA, Nathalie Gisabo (2001). La paix au féminin, chronique produite par Carrefour Tiers-Monde.

GESLIN, Jean-Dominique (2002). «Un conflit coûteux, un bilan lourd, Jeune Afrique», Lintelligent, 4-10 mars 2002.

HAUT COMMISSARIAT DES NATIONS UNIES POUR LES RÉFUGIÉS (2002). Refugees, New York.

HUMAN RIGHTS WATCH (2002). La guerre dans la guerre. Violence sexuelle contre les femmes et les filles dans l'est du Congo, juin 2002.

KELLENBERGER, Jakob (2001). L'action humanitaire dans les conflits armés actuels: contexte et besoins, Exposé fait à Zurich le 25 janvier 2001 lors du «Dolder-Meeting » organisé par le Tages-Anzeiger.

KHAN, Jooneed (2002). "Le pillage de la République démocratique du Congo », $L a$ Presse, Montréal, 24 octobre 2002.

NDUWIMANA, Françoise (2002). «Une enfance interdite par le SIDA», Le Devoir, Montréal, 3 décembre.

NDUWIMANA, Françoise (2001). «Femmes africaines face au SIDA: comment interpréter le silence?», Le Devoir, Montréal, $1^{\text {er }}$ décembre.

OLLIVIER, Émile (1991). Passages, Montréal, Hexagone.

ONUSIDA/OMS (2002). Le point sur l'épidémie du SIDA : décembre 2002.

PHYSICIANS FOR HUMAN RIGHTS (2002). War Related Sexual Violence in Sierra Leone, janvier.

Programme des Nations Unies pour le développement (2002). Rapport mondial sur le développement humain: Approfondir la démocratie dans un monde fragmenté.

SMILLIE, Ian, Lansana GBERIE et Ralph HAZLETON (2000). Le coeur du problème. Les diamants et la sécurité humaine, rapport d'enquêtes produit par Partenariat Afrique Canada, janvier.

TOUGAS, Denis (2000). «À qui profite la guerre en RDC? », Relations, octobre-novembre.

WARNER, Keith Q. (dir.) (1971). Voix françaises du monde noir, Holt, Rinehart and Winston.

\section{Note}

1. Selon le Nouveau Partenariat pour le développement de l'Afrique, octobre 2001. 